\title{
Technology Supports for Community-Dwelling Frail Older Adults
}

\author{
Richelle Stanley *
}

The University of Victoria

rstanley@uvic.ca

\begin{abstract}
Population aging is placing extensive pressure on home care programs to provide the necessary services for complex care clients to remain in their homes and avoid institutionalization or hospitalization. Finding innovative and cost-effective ways of meeting the healthcare needs of older adults and the healthcare workers who support them is becoming a pressingly urgent issue. Assistive in-home technologies, such as tools for fall prevention and medication management, have been demonstrated to positively impact health outcomes and the quality of life of autonomous older adults living in the community. This literature review identifies supportive technologies that may improve the home care of complex older adults while reducing caregiver stress through a comprehensive examination of current literature in the field of "gerontechnology." Past research has demonstrated the adoption of technology for home care support is beneficial in retaining older adult independence while reducing the risk of falls, medication errors, and caregiver stress. The findings of this review highlight how technologies are currently being integrated into home care and how the healthcare system can better include these technologies for home care support in the care of community-dwelling older adults.
\end{abstract}

Keywords: Technology; elderly; aging; home care; smart homes; aging in place

\section{INTRODUCTION}

$\mathrm{P}$ opulation aging is occurring around the globe - the numbers and proportions of older adults are rising in all countries. According to the United Nations, the global proportion of adults age 60 and over will rise from $11.7 \%$ in 2013 to $21.1 \%$ by 2050 and it is estimated that by 2050 over 2 billion people will be aged 60 and older (United Nations, Department of Economic and Social Affairs, Population Division, 2013). The most recently available data show that Canada's older adult population increased by 14.1\% between 2006 and 2011, a trend which is expected to continue in coming years (Statistics Canada, 2011). In addition to an increasing aging population, current life expectancy at birth in Canada is also increasing. Currently, the life expectancy in Canada is 81.24 years of age and is expected to increase to 86 years by 2050 (United Nations, Department of Economic and Social Affairs, Population Division, 2013). Among the growing populations of older adults, the most rapidly growing subgroup are those aged 85 and older; the age group at greatest risk for multiple comorbidities, cognitive impairment, and functional limitations that can threaten independence. Because older adults have higher utilization of healthcare services, including hospitalization, than younger age groups, home care programs are vital to allow older adults to successfully age in their communities and in their own homes where they prefer to be (Gitlow, Eastman, Gefell, O'Connor, \& Spangenberg, 2012; Mahmood,

\footnotetext{
*This literature review was made possible by the contributions and support of the Technology Evaluation in the Elderly Network.
} 
Yamamoto, Lee, \& Steggell, 2008). Certainly, the aging population has placed increased pressure on home care programs that offer the support and services to help older adults remain in their homes and prevent institutionalization.

Given both a rapidly growing population of older adults and longer life expectancies, our healthcare system is now presented with the challenge of how to provide adequate care and services. Innovative and cost-effective ways to meet the healthcare needs of older adults at home is an urgent issue. One promising strategy to meet this growing demand is to develop integrated technology systems that can supplement and even potentially replace selected caregiving services. Recent advances in "gerontechnology" have focussed on designing technologies to improve the lives of the elderly and are changing the possibilities for assessing and supporting older adults in their homes (Demiris \& Hensil, 2008; Rantz et al., 2013). The development of integrated information and communication systems to provide support will revolutionize home care. The purpose of this scoping literature review is to explore technologies being used in home care, to identify gaps in support and services, and to make recommendations for next steps in research and development in home care technologies.

\section{Purpose}

According to Reeder, Demiris and Marek (2003), the demand for home care has reached an all-time high as that provider workforce is also aging and dwindling in numbers. Appropriate technologies have the potential to reduce the workload of healthcare workers, while allowing earlier identification and treatment of symptoms, enhance coordination of care resulting in better health outcomes and improved quality of life for older adults living in the community (Jacelon \& Hanson, 2013). This scoping literature review will identify technologies currently being used in home care that may mitigate risk and improve health outcomes for older adults. Critical analysis of the literature is useful in determining how technologies are being used and whether they: 1) are effective in enhancing communication between healthcare professionals and patients, and 2) empower patients and families to manage care more effectively and independently. The results of this study are discussed in relation to the International Resident Assessment Instrument's (interRAI) home care assessment system to consider whether integrated technology might be used to inform and supplement clinical assessment (InterRAI. 2007). Lastly, the result of this review highlights the direction in which further research efforts should be focused.

\section{METHOD}

An extensive search of studies published since 2004 was conducted using automatic searches in the Cumulative Index to Nursing and Allied Health Literature (CINAHL), PubMed, Google Scholar, and Science Direct databases. Key search words and phrases used in varying combinations were "technology," "elderly," "aging," "home care," "smart homes," and "aging in place." The inclusion criteria were a) the article highlighted technologies currently being used in home care to support senior independence, and b) the article aided in the identification of new technologies being implemented in home care, and c) the article was written within the last 10 years. Articles were excluded if they did not provide sufficient information regarding the functionality and impact of technologies in home care. From this information, a total of 43 articles were read and analyzed, however only 23 were included in this review as they best met the inclusion criteria. Although there were additional articles that discussed technologies and home care, some were not included in this literature review as they were not descriptive enough in regards to the technologies being discussed or were not included in order to avoid repetition of technologies. 


\section{Results}

Technologies were classified into four common categories identified in the literature: 1) medication management and optimization, 2) fall prevention, 3) safety and behaviour monitoring, and 4) smart home technology. The majority of technologies fit into one of these categories.

\section{Medication Management and Optimization}

According to Reeder, Demiris, and Marek (2003), over $60 \%$ of older adults have at least one chronic condition requiring medication management, and on average require eight medications varying in dose, timing, and route of administration. Medication errors in seniors are a common cause of adverse events, such as hospitalization or institutionalization. In order to decrease medication errors, numerous studies indicate that automated medication management systems for older adults are effective in helping them manage complex medication regimes (Reeder et al., 2013; Marek et al. 2013). Older adults living at home without a medication management system are almost three times more likely to be admitted into a nursing home compared to those who have some kind of medication management stystem (Reeder et al., 2013). Thus, the use of automated medication systems to help older adults manage complicated medication regimes can ensure adherence and avoid medication errors.

Automatic medication dispensers in the home are an increasingly common strategy for managing medications (Reeder et al., 2013). Recent clinical trials have investigated whether the integration of an automated medication dispensing system could improve medication adherence and ultimately older-adult health outcomes (2013). The dispensing system used in these trials was called MD.2@ (Automatic monitored medication dispenser) and is commercially available. The MD.2® dispenses pre-loaded medications automatically (2013) and provides verbal alerts when medications need to be taken. In the case where medication has been missed or if the dispenser needs to be refilled, the MD.2® automatically notifies a specified contact via a phone line (Reeder et al., 2013; Marek et al. 2013). The dispenser can hold enough medications for two weeks before it needs to be refilled. The findings indicated that older adults found the dispenser easy to use, reliable, and helpful in medication management (Reeder et al. 2013). Overall, the adoption of this medication management technology helped older adults maintain independence while reducing the risk of medication error or non-adherence.

In addition to studies that investigated technology for medication dispensing, Cheek, Nikpour, and Nowlin (2005) examined information and communication technologies (ICT ) to assist with monitoring vital signs and other physiological measurements. The tools reviewed included a talking glucose meter or blood pressure and pulse monitor to provide information to adjust diabetic or hypertensive medications to reduce acute risks (2005). Another medication management technology identified was the medication sensor pad. Medications are placed on the pad, which sends an alert to the caregiver when they have been removed and taken by the client. Additional medication management technologies being developed to support home care include videoconference calls between nurses and patients to assist and remind clients to take medications, as well as assistive robots that aid the client in adhering to the medication regime (2005). These robots might take the form of a virtual pet that reminds clients to take medications and becomes ill if the medication regime is not followed.

Medication mismanagement is a pressing issue that adversely impacts health care systems and the health of seniors. In the United States alone, the annual cost of medication non-adherence is estimated at 300 billion dollars (DiMatteo, 2004). In Canada, a study discussing polypharmacy in seniors determined that $25 \%$ of patients with chronic conditions reported having an adverse 
drug event related to complex medication regimes (Canadian Institute for Health Information, 2010). Medication management technologies can be cost-effective by reducing medication errors, non-compliance, and hospitalizations, as well as reducing caregiver stress regarding complex medication regimes.

\section{Fall Prevention and Monitoring}

In addition to medication errors, falls are another risk for older adults living at home. On average, one in three older adults falls each year (Tinetti, Gordon, Sogolow, Lapin, \& Bradley, 2006). While most falls do not result in injury, a significant number result in hospitalization or other medical care. About $40 \%$ of older adults admitted to a hospital after a fall are subsequently admitted into a long term care institution and do not return home (2006). These statistics drive fall-prevention efforts and technological strategies for the home. Tchalla et al. (2012) explored whether an automated light path leading from the bedroom to the bathroom could reduce the risk of falls at night. This technology was coupled with tele assistance services so if a fall should occur, harm could be drastically reduced due to a quick response (2012). Findings indicate that the combined technologies had a significant impact on the prevention of falls at home, while early detection also reduced the likelihood of hospitalisation (2012).

Several popular approaches have incorporated automatic lighting systems (e.g. the Clapper $\mathbb{R}$ ) in which clapping activated a light) to prevent falls in the home. Advances in technology have made automated motion detecting lights a reality, as well as pressurized mats that remotely turn on the lighting system when stood on (Wagner et al., 2012).

In addition to lighting systems designed to prevent falls, a range of fall monitoring and notification systems are also being explored. A current example is a video surveillance system that uses a 3D camera programmed to track the movements of the older adult (Wagner et al., 2012). It recognizes movements suggesting a fall and sends an alert to caregivers or a response centre (2012). This strategy can be more effective than a medical alert system that requires the older adult to push a button when an emergency occurs (2012). However, continuous monitoring (even when videos are not involved) may make a client uncomfortable. A less invasive fall monitoring system is a floor vibration-based fall detector that is reasonably cost efficient (2012). This technology requires a small sensor to be installed in each room of the house that can detect the difference between an object being dropped versus a person falling. When a fall is detected, an alert is sent to a caregiver's phone or to a pre-determined response centre. At present, many of these fall detection technologies are relatively new, and more research is needed to assess their effectiveness at reducing falls and injury, as well as cost efficiency.

\section{Behaviour and Safety Monitoring}

Medical/emergency alert systems and home sensor systems (e.g., CareLink () have been available to older adults for a number of years (CareLink Advantage, 2009). For many home sensor systems, the client need only press a button and a response center will be notified of their emergency, giving clients and their caregivers a sense of security (Wagner et al., 2012). Other behaviour monitoring tools include home sensor or warning systems to alert caregivers to the absence of activities (Blaschke, Freddolino, \& Mullen, 2009). Sensors are placed in common areas of the house, such as the refrigerator, stove, or dresser drawers, and function to monitor movement. Alerts are sent to caregivers when no motion is detected after a specified period of time (Wagner, Basran, \& Bello-Hass, 2012). These sensor systems are useful for monitoring clients with dementia who are prone to wandering or for detecting changes or decline in functional levels (Blaschke, Freddolino, \& Mullen, 2009). Mattress sensors are being developed that monitor restlessness or 
getting out of bed, vital sign measurements, and body fluid leaks, like incontinence (Wagner, Basran, \& Bello-Hass, 2012). Other technologies in development to promote home safety include automatic taps where water flow is initiated via motion sensor to prevent overflow if a senior forgets to turn off the tap (Kim, Lee, \& Yim, 2009). Similarly, an intelligent bathtub system will have tap controls to limit the temperature and prevent scalding along with water flow regulators to prevent overfilling (2009). Finally, new clothing materials will have the ability to monitor heart rhythm and vital signs as well as provide global positioning satellite (GPS) location (Wagner, Basran, \& Bello-Hass, 2012). These are all relatively new types of technologies and thus have minimal testing and information regarding the benefits to older adults in the community. Further research will need to be done in this area in order to determine the effectiveness of these devices in improving older adult independence.

\section{Smart Home Technology}

One of the most comprehensive strategies emerging is the "smart home". These environments integrate specific monitoring technologies into homes to allow remote and intelligent monitoring (Demeris \& Hensel, 2009). Intelligent monitoring systems "learn" the normal behavior and patterns of an elderly individual to determine behavioural abnormalities and functional decline (Wagner, Basran, \& Bello-Hass, 2012). For example, Wagner, Basran, and Bello-Hass discuss the Intel-GE Care Innovations QuietCare system, a home monitoring and emergency alert system that utilizes smart sensors and analyzing software to detect trends and changes in the normal routine of the adult (2012). A daily report of the individual's activities is generated and sent to the caregivers to provide information such as if the client got out of bed, how many times they used the washroom, or if they used the stove to prepare a meal. The authors note that this integrated system can also detect emergencies like falls or extreme changes in function (2012). This was an example of a smart sensor system while there are projects that have created an entire smart home environment from these types of sensor systems. An example of a smart home innovation is that of the TigerPlace project in Missouri, USA (Demeris \& Hensel, 2009). This independent retirement community was designed to research specific monitoring technologies for older adults. The design of TigerPlace reflects the preferences of older adults; thus, the technologies are unobtrusive, reliable, user-friendly, detect a range of emergencies, and require minimal action from the user (Rantz et al. 2013). At TigerPlace, inexpensive motion sensors are installed throughout residences to monitor changes in activity and increase safety. Sensors can detect whether a stove is left on and bed sensors provide data on restlessness and changes in body temperature (Rantz et al., 2013). There are a multitude of other smart home systems currently being developed and tested, like the PROSAFE $R$ monitoring system in France, which detects patterns of function (Wagner, Basran, \& Bello-Hass, 2012). The AwareHome project in Georgia tests emerging home technologies and introduced tracking technology to help older adults keep track of commonly lost items such as wallets, glasses, and keys (2012).

Many of these technologies are still in the early stages of development and more research is needed to evaluate cost-effectiveness and impact on outcomes. However, the potential benefit of smart-home technology on older adults is significant. TigerPlace researchers have shown that sensors detect changes in function in elders two weeks before significant health events like falls or illness occurred (Rantz et al. . 2013). Home technologies can support older adults in the home, allowing aging in place to be a reality for seniors, thus significantly improving quality of life by increasing safety (2013).

The table below collectively organizes the many technologies introduced in this review into the four previously highlighted categories of types technologies used in home care. 


\section{MEDICATION MANAGEMENT}

These kinds of technologies were found to be cost effective by reducing medication errors, non-compliance, hospitalization, and caregiver burden

- Automated Medication dispenser (MD.2)®

- Assistive monitoring tools with voice prompts - e.g. blood pressure, pulse, and blood glucose

- Medication sensor pad to notify caregiver if medication not taken

- Video conference calls with home care nurse for medication reminders

\section{BEHAVIOUR AND SAFETY MONITORING}

These home-tools are designed to provide clients and caregivers with a substantial sense of security

- Medical/emergency alert systems that are client activated through personal device (e.g. Carelink)

- Home and furniture sensors to detect client level of function

- Automated faucets to control water temperature and overfill

- Technology-fitted clothing to monitor vital signs and provide GPS location

\section{FALL PREVENTION}

Many older adults who experience a fall will be hospitalized or institutionalized and will not return to their home in community; these technologies have been shown to help reduce that risk

- Automated light path guides client from bed to bathroom - coupled with teleassistance

- Automated motion detecting lights

- Pressurized mats turn lights on when stood on

- Video Surveillance coupled with teleassistance

- Floor vibration-based fall detector

SMART HOME TECHNOLOGY

Smart home intelligent monitoring systems "learn" the normal behaviour and pattern of the elderly individual to determine behavioural abnormalities and functional decline

- The QuietCare home monitoring system utilizes smart sensors and analyzing software to detect functional changes

- Home and furniture sensors to detect client level of function

- TigerPlace is a retirement community designed to research specific monitoring sensors and technologies to detect changes and increase safety

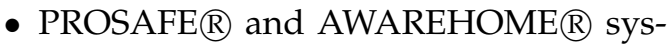
tems are in development to detect patterns of function 
The Arbutus Review • Fall $2015 \bullet$ Vol. 6, No. 1

\section{Discussion}

This literature review examines some of the most promising technologies that are currently commercially available or being developed for home care purposes. The research clearly demonstrates that home technology strategies can reduce or delay institutionalization while maintaining quality of life and increasing safety. Although home technologies have consistently shown to improve health outcomes, there are many barriers that prevent it from being readily adopted, such as financial limitations and a lack of knowledge about technologies. In terms of financial limitations, these home technologies and home modifications remain relatively inexpensive, offering a costeffective alternative to long-term residential facilities (Tomita, Mann, Stanton, Tomita, \& Sundar, 2007). Despite this, more research is needed to determine if technology for home care support is a realistic possibility for those with limited finances (Boger, Quraishi, Turcotte, \& Dunal, 2014).

Currently, technology is utilized minimally in home care, for reasons that include limited caregiver knowledge about technologies and the lack of healthcare coverage for it. For example, there was a recent study conducted by Boger et al.(2014) that focused on the opinions of caregivers who discussed the kinds of tools they thought would be useful to reduce stress and workload in caring for older adults. Many of the technologies suggested already existed, but the general population was not aware of common technologies like medication dispensers or fall monitoring systems (Boger, Quraishi, Turcotte, \& Dunal, 2014). Clearly, more information regarding commercially available products needs to be provided to caregivers.

One method that might increase awareness of available technologies may be through the implementation of an assessment system that uses eligibility criteria to gauge health and care needs and recommend appropriate technologies to benefit older adults (Demiris \& Hansel, 2009). An example of a system currently being used in Canada is the home care InterRAI assessment instrument, which was designed to highlight and address issues in functioning and quality of life in community-dwelling seniors (InterRAI, 2009). Home care nurses use this comprehensive system to assess the needs, strengths, and preferences of a client through consideration of activities of daily living, pain, and mental status. The InterRai assessment is administered in person and used to determine what home care supports are needed for the client and to monitor changes in health and function for home care services (InterRAI, 2009). These scores are then analyzed by the software system that indicates areas of the care plan that may need changes since the last assessment. For example, the software will highlight if the client is at risk for institutionalization and if so, what areas of the care plan need to be addressed in order to reduce this risk.

This scoping review suggests that this type of assessment system may also be a tool to determine which technologies a client may benefit from based on their domain scores. For example, a client at risk for institutionalization due to poor medication management scores would be identified by the system as an individual who may benefit from trialing an assistive medication device. Likewise, if an InterRai assessment discovers a client has a high risk of falling, it would indicate that the client may benefit from a fall prevention device.

Although these technologies have been shown to be cost-effective in the long run, the biggest limitation of this review remains as difficulty in determining the cost-effectiveness of initially integrating supportive technology into home care. There remains the question of who pays for the initial start-up costs as well as the cost of maintenance of technology systems. As well, it is unclear if reliance on these home systems may mask early cognitive or function decline, thus delaying health diagnoses (e.g., dementia, Parkinson's). However, assistive at-home tools may still be high-gain and low-cost: their long term benefits to older adult health outcomes, quality of life, caregiver stress, and healthcare system expenses may ultimately outweigh their limitations. Because many of the technologies discussed in the literature are still under development, it will 
take a significant amount of time and continued research efforts before they become commercially available and aid in making prolonged community-dwelling a reality. The purpose of this review was to determine if the adoption of home care technologies could assist older adults in remaining in their homes in the community. It was ultimately determined that while current research has shown the beneficial impact of home care technologies on seniors, further research efforts need to move toward to cost effectiveness and overall development and testing of these technologies.

\section{Conclusion}

In 2009, almost 80,000 seniors in Canada indicated that they had at least one unmet need for their home care services (Hoover \& Rotermann, 2012). As the number of seniors in Canada increases, the ability of home care workers to meet these needs within our current care system is going to become increasingly difficult. There are many opportunities to integrate supportive technology into home care which, despite current questions of cost-effectiveness, may be a viable way to reduce the stress on home care services and caregivers, while enabling older adults to make the most out of their independence and lives in the community. This review has highlighted several types of technologies that are currently in use or being developed and provided insight into methods of integrating these technologies into the lives of community-dwelling older adults. It is hoped that future research efforts can better highlight the overall cost effectiveness of in home technology systems for older adults, as well as determine the kinds of technologies that older adults and their families indicate as beneficial to improving quality of life in the home.

\section{REFERENCES}

Blaschke, C., Freddolino, P., \& Mullen, E. (2009). Ageing and technology: A review of the research literature. British Journal of Social Work, 39(4), 641-656. http://dx.doi.org/10.1093/bjsw/bcp025

Boger, J., Quraishi, M., Turcotte, N., \& Dunal, L. (2014). The identification of assistive technologies being used to support the daily occupations of community-dwelling older adults with dementia: a cross-sectional pilot study. Disability and Rehabilitation: Assistive Technology, 9(1), 17-30. http://dx.doi.org/10.3109/17483107.2013.785035

Cheek, P., Nikpour, L., \& Nowlin, H. D. (2005). Aging well with smart technology. Nursing administration quarterly, 29(4), 329-338. http://dx.doi.org/10.1097/00006216-200510000-00007

Demiris, G., \& Hensel, B. (2009). "Smart homes" for patients at the end of life. Journal of Housing for the Elderly, 23(1-2), 106-115. http://dx.doi.org/10.1080/02763890802665049

Demiris, G., \& Hensel, B. K. (2008). Technologies for an aging society: a systematic review of "smart home" applications. Yearb Med Inform, 3, 33-40.

DiMatteo, M. (2004). Evidence-based strategies to foster adherence and improve patient outcomes. JAAPA: official journal of the American Academy of Physician Assistants, 17(11), 18.

Gitlow, L., Eastman, E., Gefell, A., Morehouse, L., O'Connor, C., \& Spangenberg, C. (2012). Assessing assistive device needs of community dwelling older adults in Tompkins County. Physical Eamp; Occupational Therapy In Geriatrics, 30(4), 368-382. http://dx.doi.org/10.3109/02703181.2012.736125

Hoover, M. \& Rotermann, M. (2012). Seniors' use of and unmet needs for home care. Statistics Canada Health Reports Division. 
InterRAI. (2007). Home care assesment system. Retrieved from http://www.interrai.org/home-care . $\mathrm{html}$

Jacelon, C. S., \& Hanson, A. (2013). Older adults' participation in the development of smart environments: An integrated review of the literature. Geriatric Nursing, 34(2), 116-121. http://dx.doi.org/10.1016/j.gerinurse.2012.11.001

Kim, S.-K., Lee, Y.-M., \& Yim, M.-S. (2009). High-tech amenities for the elderly: The technological assistance needs of elderly Koreans aging at home. Journal of Housing for the Elderly, 23(3), 204-226. http://dx.doi.org/10.1080/02763890903035548

Mahmood, A., Yamamoto, T., Lee, M., \& Steggell, C. (2008). Perceptions and use of gerotechnology: implications for aging in place. Iournal of Housing for the Elderly, 22(1-2), 104-126. http://dx.doi.org/10.1080/02763890802097144

Marek, K. D., Stetzer, F., Ryan, P. A., Bub, L. D., Adams, S. J., Schlidt, A., .. O'Brien, A.-M. (2013). Nurse care coordination and technology effects on health status of frail elderly via enhanced self-management of medication: Randomized clinical trial to test efficacy. Nursing research, 62(4), 269. http://dx.doi.org/10.1097/nnr.0b013e318298aa55

Rantz, M. J., Skubic, M., Miller, S. J., Galambos, C., Alexander, G., Keller, J., \& Popescu, M. (2013). Sensor technology to support aging in place. Journal of the American Medical Directors Association, 14(6), 386-391. http://dx.doi.org/10.1016/j.jamda.2013.02.018

Reeder, B., Demiris, G., \& Marek, K. D. (2013). Older adults' satisfaction with a medication dispensing device in home care. Informatics for Health and Social Care, 38(3), 211-222. http://dx.doi.org/10.3109/17538157.2012.741084

Tchalla, E., Lachal, F., Cardinaud, N., Saulnier, I., Bhalla, D., Roquejoffre, A., ... Dantoine, T. (2012). Efficacy of simple home-based technologies combined with a monitoring assistive center in decreasing falls in a frail elderly population (results of the Esoppe study). Archives of gerontology and geriatrics, 55(3), 683-689. http://dx.doi.org/10.1016/j.archger.2012.05.011

Tinetti, M. E., Gordon, C., Sogolow, E., Lapin, P., \& Bradley, E. H. (2006). Fall-risk evaluation and management:challenges in adopting geriatric care practices. The Gerontologist, 46(6), 717-725. http://dx.doi.org/10.1093/geront/46.6.717

Tomita, M. R., Mann, W. C., Stanton, K., Tomita, A. D., \& Sundar, V. (2007). Use of currently available smart home technology by frail elders: Process and outcomes. Topics in geriatric rehabilitation, 23(1), 24-34. http://dx.doi.org/10.1097/00013614-200701000-00005

United Nations, Department of Economic and Social Affairs, Population Division. (2013). World population ageing. Retrieved from http://www.un.org/en/development/desa/population/ publications/pdf/ageing/WorldPopulationAgeing2013.pdf

Wagner, F., Basran, J., \& Dal Bello-Haas, V. (2012). A review of monitoring technology for use with older adults. Journal of geriatric physical therapy, 35(1), 28-34.

http://dx.doi.org/10.1519/jpt.0b013e318224aa23 